in Developmental Disabilities

Elsevier Editorial System(tm) for Research

Manuscript Draft

Manuscript Number: RIDD-D-12-00577R1

Title: Developmental Dyscalculia and Low Numeracy in Chinese Children

Article Type: Research Paper

Keywords: dyscalculia; low numeracy; mathematical achievement; symbolic processing; non-symbolic processing.

Corresponding Author: Dr. Winnie Wai Lan Chan,

Corresponding Author's Institution:

First Author: Winnie Wai Lan Chan

Order of Authors: Winnie Wai Lan Chan; Terry K. Au; Joey Tang

Abstract: Children struggle with mathematics for different reasons. Developmental dyscalculia and low numeracy - two kinds of mathematical difficulties - may have their roots, respectively, in poor understanding of exact non-symbolic numerosities and of symbolic numerals. This study was the first to explore whether chinese children, despite cultural and linguistic factors supporting their mathematical learning, also showed such mathematical difficulties and whether such difficulties have measurable impact on children's early school mathematical performance. First-graders, classified as dyscalculia, low numeracy, or normal achievement, were compared for their performance in various school mathematical tasks requiring a grasp of non-symbolic numerosities (i.e., non-symbolic tasks) or an understanding of symbolic numerals (i.e., symbolic tasks). Children with dyscalculia showed poorer performance than their peers in non-symbolic tasks but not symbolic ones, whereas those with low numeracy showed poorer performance in symbolic tasks but not non-symbolic ones. As hypothesized, these findings suggested that dyscalculia and low numeracy were distinct deficits and caused by deficits in non-symbolic and symbolic processing respectively. These findings went beyond prior research that only documented generally low mathematical achievements for these two groups of children. Moreover, these deficits appeared to be persistent and could not be remedied simply through day-to-day school mathematical learning. The present findings highlighted the importance of tailoring early learning support for children with these distinct deficits, and pointed to future directions for the screening of such mathematical difficulties among Chinese children. 


\title{
Developmental Dyscalculia and Low Numeracy in Chinese Children
}

\author{
Winnie Wai Lan Chan ${ }^{\mathrm{a}}$, Terry K. Au ${ }^{\mathrm{a}}$, Joey Tang ${ }^{\mathrm{a}}$ \\ ${ }^{a}$ Department of Psychology, University of Hong Kong, Hong Kong SAR, China \\ Corresponding author: Winnie Wai Lan Chan \\ Email: winniewinniec@gmail.com \\ Phone: +85291271306
}




\section{Introduction}

Mathematics literacy is important for survival in modern society. Everyday tasks such as paying bills or budgeting for daily expenses all have to do with numbers. Most children first learn numeracy at preschool, but some already fall behind their peers in mathematics by first grade. Previous studies (Butterworth, 2005a; Shalev, 2007) have estimated that $6-7 \%$ of children suffer from developmental dyscalculia (DD), a learning difficulty in mathematics characterized by problems in acquiring and remembering arithmetical facts (Geary \& Hoard, 2001; Jordan, Hanich, \& Kaplan, 2003; Shavlev \& Gross-Tsur, 2001) as well as executing calculation procedures (Geary, 1993).

One account for developmental dyscalculia focuses on a specific deficit in basic numerical processing (Landerl, Bevan, \& Butterworth, 2004). According to this account, namely the number module theory (Butterworth, 1999, 2005a), children are born with a specific core numerical capacity for representing and processing numerical information, which is governed by specialized neural network dissociable from non-numerical capacities such as language (Cohen, Dehaene, Cochon, Lehericy, \& Naccache, 2000) and working memory (Butterworth, Cipolotti, \& Warrington, 1996). With such innate numerical capacity, even infants can discriminate small numerosities (Starkey \& Cooper, 1980; Starkey, Spelke, \&Gelman, 1990; Wynn, 1992, 1995). This capacity is derived from a "number module" constructing concepts of exact numerosities of sets, which lays the foundation for arithmetic 
development (e.g., Butterworth, 2005b; Butterworth \& Reigosa-Crespo, 2007). A deficit in this core numerical capacity underlies developmental dyscalculia, according to the "defective number module hypothesis" (Butterworth, 2005a).

Due to an inability to represent exact numerosities, children with developmental dyscalculia tend to be slow and inefficient in even very basic numerical tasks, such as recognizing and comparing the numerosities of object sets (Butterworth, 2005a; Landerl et al., 2004; Inculano, Tang, Hall, \& Butterworth, 2008). Yet, they can still perform well in non-numerical academic subjects and have normal or even superior intelligence (Butterworth, 2005a; Landerl et al., 2004). Dyscalculia Screener (Butterworth, 2003) is a standardized software with an English norm for screening dyscalculic children. Children who are classified as dyscalculic should perform substantially below average in at least one of the two tasks tapping basic numerical processing, namely recognizing the numerosities of displays of dots (i.e., dot enumeration task) and comparing the numerosities of numbers (i.e., number comparison task).

However, not all of the children who struggle with mathematics are dyscalculic. Some of them have intact concept of exact numerosities, but poor understanding of symbolic numerals. These children are hypothesized to have low numeracy, a mathematical difficulty caused by problem in relating symbols to the concept of numerosities (Iuculano et al., 2008). Unlike dyscalculic children, they perform at normal level in non-symbolic tasks such as 
comparing the numerosities of objects (Rousselle \& Noël, 2007). Yet, they fail when the tasks involve numerical symbols (i.e., symbolic tasks), such as adding numerals (Rousselle \& Noël, 2007). On the Dyscalculia Screener (Butterworth, 2003), children with low numeracy should perform within normal range on the two tasks tapping basic numerical processing, but show significantly impaired performance in the (symbolic) addition task (Iuculano et al., 2008).

While prior research has only documented generally poor mathematical performance for children with dyscalculia or low numeracy, it remains unclear if, and how, these two supposedly distinct deficits may impact on children's mathematical learning and achievement differently. In this study, we examined how the underlying deficits in developmental dyscalculia and low numeracy would differentially affect children's performance in various numerical tasks during their early formal mathematical learning in the elementary school. Our findings can enhance teachers' and parents'awareness and understanding of these children's difficulties in acquiring school mathematics, thereby facilitating early screening for appropriate learning support. The tasks adopted in this study were designed to assess various targeted numerical skills in the first grade (Hong Kong Curriculum Developmental Council, 2000), such as reciting count names, place-value concept, and simple addition. These tasks were further categorized as symbolic, involving numerical symbols, or non-symbolic, involving pictorial objects instead of numerals. If developmental dyscalculia 
is indeed caused by a deficit in processing exact non-symbolic numerosities whereas low numeracy is caused by a deficit in symbolic numerical processing, children with dyscalculia should show impaired performance in non-symbolic tasks but not symbolic ones, while children with low numeracy should have impaired performance in symbolic tasks but not non-symbolic ones. To see whether the underlying deficits persist over time or can be compensated by day-to-day school learning, we traced the children's numerical performance across the two semesters in the first grade.

This study is also the first to explore developmental dyscalculia and low numeracy among Chinese children. To date, the findings and theories on mathematical difficulties are primarily based on studies with Western children. Fundamental questions remain open. First, how robust are these findings and theories across cultures and languages? Consider Chinese children as a case in point: Academic achievements in general, and mathematical skills in particular, are highly valued in the Chinese culture. Children are taught to count and add and subtract with the Arabic number system from an early age, with massive practices integrated in daily routines and play (Huntsinger, Jose, Liaw, \& Ching, 1997; Zhou et al., 2006). Indeed, informal numerical abilities are more developed in 4- to 5-year-old Chinese as well as other East Asian (Japanese and Korean) children than their counterparts in the U.S. (Starkey \& Klein, 2008).

Moreover, the number naming system in Chinese is highly regular and transparent (e.g., 
the count name for " 11 " in Chinese literally means "ten-one," unlike eleven in English). In terms of information processing, all single-digit numbers in Chinese are labeled with single syllables with simple phonological structure (i.e., consonant-vowel or consonant-vowel-consonant), whereas the name for "7" in English has two syllables, and the names for " 3 " and "6" in English have tricky consonant clusters (i.e., the amalgam of the "th" and " $\mathrm{r}$ " sounds in three and the amalgam of " $\mathrm{k}$ " and "s" sounds for six.) Indeed, Chinese preschool children's counting abilities are much more precocious than those of their English-speaking counterparts (Miller, Smith, Zhu, \& Zhang, 1995; Miller \& Stigler, 1987). Such cultural difference occurs even at age 3 and increases during childhood (Miller, Kelly, \& Zhou, 2005). With cultural emphasis and a user-friendly number naming system on their side, are dyscalculia and low numeracy problems for Chinese children? If yes, how might such difficulties be manifested in their mathematical learning and tasks performance?

There is a growing concern over how theories and research findings from Western cultures can be applied to Chinese children in order to understand and accommodate their difficulties in mathematical learning. Our findings would bridge the gap between East and West in the study of dyscalculia and low numeracy, and provide implications for the direction of future investigation into mathematical difficulties among Chinese children.

\section{Method}




\subsection{Participants}

Seventy-four Grade 1 children were recruited from 18 primary schools and categorized into three groups: developmental dyscalculia group (DD, $N=28)$, low numeracy group (LN, $N=9$ ), normal achievement group (NA, $N=37$ ). They were selected by screening a sample of 495 children from another study. All of these 74 children scored 80 or above on the short form of Raven's Standard Progressive Matrices with a local norm and were confirmed by their parents to have never been diagnosed with or suspected of dyslexia. All children were native speakers of Chinese.

\subsection{Selection Procedures}

Children from the selection pool $(\mathrm{N}=495)$ completed a web-based test battery (Tang, 2010), which was modeled on the Dyscalculia Screener developed in the U.K. (Butterworth, 2003). The test battery consisted of: (1) two simple reaction time tasks for controlling the general response time; (2) a capacity subscale that included a number comparison task and a dot-number matching task; (3) an achievement subscale based on an addition task. As with the Dyscalculia Screener, this web-based screener presented all numbers in Arabic format the conventional number system used by both Chinese and Western children from early on.

For each task in this web-based screener, children went through four practice trials (with feedback) before doing four warm-up trials and then the experimental trials. The children were allowed to repeat the practice trials as many times as possible until they got at 
least three correct. Both the practice and warm-up trials would not count towards the median reaction time and overall accuracy of the tasks. Calculation of the median reaction time was based on the correct trials only.

\subsubsection{Simple Reaction Time Tasks}

These tasks measured children's motor response time for left and right hand respectively. In each task, children were asked to press a key as soon as they saw a black dot appearing randomly on the screen with a grey background. In the left-hand task, they pressed the "F" key with their left index finger; in the right-hand task, they pressed the "J"key with right index finger. There were a total of 20 trials in each task. The first four trials were warm-up trials.

\subsubsection{Capacity Subscale: Dot-Number Matching Task}

This task assessed children's capacity to represent exact numerosities and their understanding of the numerosities represented by single-digit numbers. Children were randomly assigned to either a left- or right-hand block. In the left-hand block, children were asked to indicate "yes" by pressing the "F" key with their left index finger and to indicate "no" by pressing the "J" key with their right index finger. In the right-hand block, children were asked to do the opposite: to indicate "yes" by pressing the "J" key with their right index finger and to indicate "no" by pressing the "F" key with their left index finger. In each trial, children were shown a single-digit Arabic number on one side of the screen and some dots 
scattering randomly on the other side. They were asked to decide as quickly as possible whether the numerosity of the dots matched with the Arabic number by pressing the appropriate keys.

Each trial was preceded by a fixation cross lasting for $500 \mathrm{~ms}$. Then the stimuli appeared for a maximum of $8000 \mathrm{~ms}$. There was a pause of $2000 \mathrm{~ms}$ between trials. The task included four warm-up trials and then 32 experimental trials. The numerosity of dots matched with the Arabic number in half of the trials (i.e., matched trials) and mismatched by a difference of one or two in the remaining trials (i.e., mismatched trials). Only the matched trials would count towards the median reaction time and overall accuracy.

\subsubsection{Capacity Subscale: Number Comparison Task}

This task assessed children's capacity to understand and compare the magnitude of single-digit numbers. In each trial, children were shown two single-digit numbers side by side on the computer screen and asked to decide which one was larger as quickly as possible.

If the one on the left was larger, they should press the "F" key with left index finger; if the one on the right was larger, they should press the "J" key with right index finger. Each trial was preceded by a fixation cross lasting for $500 \mathrm{~ms}$. Then the stimuli appeared for a maximum of $5000 \mathrm{~ms}$. There was a pause of $2000 \mathrm{~ms}$ between trials. A total of 36 trials including the four initial, warm-up trials were presented. The larger number appeared on the left in half of the trials and on the right in the other half. The two numbers could differ by one to four. 


\subsubsection{Achievement Subscale: Addition Task}

This task assessed children's capacity to do exact arithmetic. As in the dot-number matching task, children were randomly assigned to either the left- or the right-hand block. Response instructions were the same as those in the dot-number matching task.

In each trial, children were shown a single-digit addition problem and asked to decide as fast as possible whether the result was correct (e.g., " $3+7=10$ ") by pressing the appropriate key. Each trial was preceded by a fixation cross lasting for $500 \mathrm{~ms}$. Then the stimuli appeared for a maximum of $8000 \mathrm{~ms}$. There was a pause of $2000 \mathrm{~ms}$ between trials. A total of 20 trials including the four initial, warm-up trials were presented. Half of the trials were correct and half were incorrect. In the incorrect trials, the discrepancies between the presented, incorrect sum and the actual, correct one were one or two. The numbers 0 and 1 were never used as operands. Tie problems, such as $5+5$, were also excluded.

\subsubsection{Classification of Children (DD, LN, and NA)}

Children's performance on each task in the capacity subscale and achievement subscale was indicated by an inverse efficiency score, which was computed by dividing the adjusted median reaction time by the accuracy rate. The adjusted median reaction time was calculated by subtracting the mean of the median reaction times in the two simple reaction time tasks from the median reaction time in the task being examined. This was to control the individual differences in motor response time. The resulting inverse efficiency score could 
be interpreted as an adjusted reaction time score: the higher the score, the less efficient the performance would be.

Following the classification procedure of the Dyscalculia Screener developed in the U.K. (Butterworth, 2003), we identified 5.7\% of children as DD (28 out of 495). This was close to the previous estimate of 6-7\% prevalence rate for DD in the U.K. (Butterworth, 2005a; Shalev, 2007).

Figure 1 shows the flow chart of classification of the three groups of children.

Children from the selection sample were classified as developmental dyscalculia (DD) if their inverse efficiency scores in any one of the two tasks of the capacity subscale (i.e., number comparison task and dot-number matching task) was higher than the sample mean by at least two standard deviations (indicating a deficit). Children were classified as low numeracy (LN) if their inverse efficiency scores in the addition task of the achievement subscale were higher than the sample mean by at least two standard deviations (indicating a deficit), while their scores on both tasks in the capacity subscale were no higher than the sample mean by one standard deviation (suggesting normal performance). Children in the normal achievement (NA) group were matched one on one with children in the DD and LN groups on the basis of age, gender, nonverbal intelligence, school, and classroom as far as possible. Children in the NA group had their inverse efficiency scores in each task of the capacity and achievement subscales no higher than the sample mean by one standard deviation. Table 1 
shows the descriptive information of the children. The groups did not differ significantly in age and nonverbal intelligence (all $p \mathrm{~s}>.05$ ).

\subsection{Mathematical Achievement Assessment}

From the selection pool $(N=495), 74$ children were selected with the web-based test battery (Tang, 2010) at the end of fall semester in first grade. There were 28 children with developmental dyscalculia (DD), 9 with low numeracy (LN), and 37 with normal achievement for addition problems (NA). The selected children $(N=74)$ went on to complete a series of tasks assessing different aspects of mathematical performance: count sequence, reading aloud numbers, place-value concept, symbolic and non-symbolic addition. These tasks were administered individually to children by a research assistant. The children completed this battery of mathematical performance tasks again at the end of spring semester in first grade.

\subsubsection{Symbolic Tasks}

\subsubsection{Count Sequence}

This was to assess children's knowledge of count sequence up to 120 . In each trial, a child was given a start number and asked to count up verbally until a certain number was reached. The trials started with single-digit numbers and covered the teens, twenties, thirties, and so on, up to 120. Each trial contained a transition to a next decade (e.g., 29 to 30).

Each correct number in correct sequence would score one mark. 


\subsubsection{Reading Aloud Numbers}

Children's ability to recognize and represent verbally the written Arabic and Chinese numbers was assessed. In each task, a child was shown eight numbers in written either Arabic or Chinese and asked to read them out one by one. Half of the numbers in each task were two-digit; the other half were three-digit. One mark was given to each correct number.

\subsubsection{Symbolic Addition}

This task assessed children's ability to do exact symbolic additions. In a total of eight items, half were presented in vertical format and the other in horizontal format. In each format, half involved carry-over (e.g., $26+7$ ), and the other did not (e.g., $13+2$ ). Children were asked to compute the addition problems without time limit. Each correct answer scored one mark.

\subsubsection{Non-symbolic Tasks}

\subsubsection{Place-Value Concept}

This was assessed through three tasks, namely grouping-in-ten, place-value representation, and strategic counting.

(1) Grouping-in-ten: This task assessed children's concept of base-ten partitioning, which was a fundamental place-value component (Saxton \& Cakir, 2006). Each child was shown pictures of blocks and asked to decide how many robots - each requiring ten blocks - could be built, and how many blocks, if any, would be left over. 
There were four items. An item would score one mark if both numbers of robots and unused blocks were correct.

(2) Place-Value Representation: This task assessed children's knowledge of the quantities denoted by ones and tens place in number. In each of the four trials, each child was shown a two-digit Arabic number and asked to represent it with some base-ten magnetic small squares and bars. Each bar contained ten small squares. A child would score one mark if the child's representation corresponded to the base-ten numeration structure (e.g., "17" should be represented by a bar and seven small squares). If a child failed to construct a base-ten representation, the child would be helped to construct one in order to go on to the next part of the testing. Based on the base-ten representation, the child was then asked to make changes to it accordingly when either the digit in the ones or tens place increased. A change which corresponded to the base-ten numeration structure would score one mark (e.g., when the digit 1 in the number " 14 " increased to 2, a bar should be added). Each trial had a maximum score of two.

(3) Strategic Counting: This task was a comprehensive place-value test tapping the application and integration of various place-value components in a counting activity, such as grouping in tens, carrying over, and mapping between quantities and numerical symbols (Chan, Au, \& Tang, under review). Note that the place-value 
concept essentially connects exact numerosities with symbolic numerals. Hence in this task, so as all other place-value tasks, would inevitably involve symbolic numerals (in our task, children was asked to write down their answers in Arabic numbers; see below for details). Each of the ten items contained pictures of base-ten manipulatives, namely small squares, bars, and large squares. A bar was equivalent to ten small squares, and a large square was equivalent to ten bars or 100 small squares. Children were asked to count and write down the totals of small squares in each item in Arabic. Before starting, they were explained by an experimenter for the equivalent quantities of the manipulatives using small squares, bars, and large squares of magnets. Half of the items involved two-digit quantities, whereas the other half contained three-digit quantities. One mark was given to each correct answer.

\subsubsection{Non-symbolic Addition}

This task assessed children's ability to add objects without involving numerical symbols. In each of the four items, a child would be shown pictures of scattered blocks and whole robots, each being built with ten blocks. The child was then asked to sum up the total number of blocks including those making up the robots. Each correct answer scored one mark.

\section{Results}


Children in the three groups were compared for their raw scores in various numerical tasks given at the end of the fall and spring semesters of first grade in a series of $2 \times 3$ repeated measures ANOVAs, with semester as the within-subject factor and group as the between-subject factor. In these analyses, the Levene's tests consistently yielded nonsignificant results $(p>.05)$, indicating that the assumption of homogeneity of variance was valid even though the three groups varied in sample size. Figures $2 \mathrm{a}-2 \mathrm{~h}$ show the performance of the three groups across the two semesters in each task.

\subsection{Symbolic Tasks}

\subsubsection{Count Sequence}

None of the effects was found significant (all $p \mathrm{~s}>.05$ ), indicating that children in the three groups did not differ significantly in performance across the two semesters.

\subsubsection{Reading Aloud Arabic Numbers}

Children generally performed better after a semester, $F(1,71)=7.49, p=.008, \eta_{\mathrm{p}}{ }^{2}$ $=.10$. Significant group effect was also found, $F(2,71)=4.88, p=.01, \eta_{\mathrm{p}}{ }^{2}=.12$, where children in the LN group were significantly outperformed by their peers in the NA group ( $p$ $=.01)$. The interaction between time and group was not significant $(p>.05)$.

\subsubsection{Reading Aloud Chinese Numbers}

None of the effects reached statistical significance (all $p \mathrm{~s}>.05$ ), indicating that children in the three groups performed comparably across the two semesters. 


\subsubsection{Symbolic Addition}

A significant time effect was found, $F(1,71)=33.37, p<.0001, \eta_{\mathrm{p}}{ }^{2}=.32$, where children improved their performance over a semester. The group effect was also significant, $F(2,71)=7.01, p=.002, \eta_{\mathrm{p}}{ }^{2}=.17$, such that children in the LN group were significantly outperformed by their peers in the DD and NA groups ( $p=.04$ and $p=.001$, respectively). The group effect was further modulated by time, $F(2,71)=5.62, p=.005, \eta_{\mathrm{p}}{ }^{2}=.14$, where the inferiority of the LN group reached statistical significance in the fall $(p s<.05)$ but not spring semester.

\subsection{Non-symbolic Tasks}

\subsubsection{Grouping-In-Ten}

A significant time effect was found, $F(1,71)=5.98, p=.02, \eta_{\mathrm{p}}{ }^{2}=.08$, indicating a general improvement in performance across the semesters. Group effect was also found significant, $F(2,71)=4.01, p=.02, \eta_{\mathrm{p}}^{2}=.10$, such that children in the DD group were marginally significantly outperformed by their peers in the NA group $(p=.059)$. The interaction between time and group was not significant $(p>.05)$.

\subsubsection{Place-Value Representation}

The main effect for time was significant, $F(1,71)=6.12, p=.02, \eta_{\mathrm{p}}{ }^{2}=.08$, showing a general improvement in performance across the semesters. The main effect for group was marginally significant, $F(2,71)=2.66, p=.077, \eta_{\mathrm{p}}{ }^{2}=.07$, where children in the DD group 
were significantly outperformed by their peers in the NA group $(p=.03)$. No significant interaction effect between time and group was found $(p>.05)$.

\subsubsection{Strategic Counting}

Children in general showed better performance after a semester, $F(1,71)=21.44, p$

$<.0001, \eta_{\mathrm{p}}{ }^{2}=.23$. A significant group effect was also found, $F(2,71)=6.08, p=.004, \eta_{\mathrm{p}}{ }^{2}$ $=.15$, such that children in the DD and LN groups were significantly outperformed by their peers in the NA group ( $p=.04$ and $p=.01$, respectively). The interaction effect between time and group was not significant $(p>.05)$.

\subsubsection{Non-symbolic Addition}

None of the effects was found significant (all $p \mathrm{~s}>.05$ ), indicating that children in the three groups did not differ significantly in performance across the two semesters.

\section{Discussion}

In this study, we explored how the underlying deficits in developmental dyscalculia and low numeracy would affect children's numerical performance in early elementary school.

Children in DD, LN, and NA groups were compared for their performance in a series of symbolic and non-symbolic tasks across the two semesters of first grade. Results showed that the three groups improved significantly across the semesters on all tasks except for count sequence, reading aloud Chinese numbers, and non-symbolic addition, which indicated stable 
performance across the semesters for the three groups. Although the three groups made significant progress on most of the tasks, some groups kept lagging behind their counterparts on certain kinds of tasks.

\subsection{Symbolic Tasks}

If low numeracy is caused by a specific deficit in relating symbols to the concept of numerosities (Iuculano et al., 2008), children with such difficulty should have problem with symbolic tasks. Indeed, children in the LN group were outperformed by their peers in NA group in both semesters on reading aloud Arabic numbers. In order to read out the numbers in Chinese, children would need to have a good grasp of the numerosities represented by the base-ten symbolic system. For example, the number " 124 " is read as "one hundred two ten four" in Chinese, where "hundred" and "ten" are the underlying quantities held by the numerals in the hundreds and tens places respectively. With a deficit in associating symbols with their underlying quantities, children with low numeracy thus showed problem with reading aloud Arabic numbers.

On symbolic addition, children in the LN group were outperformed by their peers in both the NA and the DD group at the end of the first semester. This was probably due to the LN group's poor understanding of symbolic numerals. Yet, they appeared to catch up with their counterparts by the end of the second semester. This was unlikely to be due to an improvement of their ability to make sense of the symbolic numerals, given that they still had 
problem with reading aloud the Arabic numerals. Rather, they might add the numerals by falling back on strategies such as rote learning and counting on fingers to compensate for their difficulties in making sense of symbolic numerals. Future investigation into their strategies would help us understand more about how these children managed to "overcome" their difficulties in daily symbolic computation.

While both count sequence and reading aloud Chinese numbers also required the use of symbols to represent numerosities, children in the LN group appeared to perform as well as their counterparts on these tasks. One possibility was that children in the LN group fell back on strategies which did not necessarily require the association between symbols and the concept of numerosities. Since the count sequence task only required them to recite the count names in sequence without referring to their underlying numerosities, children in the LN group might simply treat the count names as a meaningless sequence and learn them by heart. Similarly, they could treat the task of reading aloud Chinese numbers as a kind of Chinese word reading task, where they just needed to read out the words as presented without even understanding the numerosities held by the numerals.

\subsection{Non-symbolic Tasks}

According to the "defective number module hypothesis" (Butterworth, 2005a), children with dyscalculia have a core deficit in processing exact non-symbolic numerosities, and would thus show difficulty on tasks involving manipulation of non-symbolic numerosities. 
Indeed, children in the DD group showed poorer performance than their peers in the NA group on grouping-in-ten, place-value representation, and strategic counting. All these tasks required children to have a sense of the exact numerosities represented by some concrete manipulatives or numerals.

On strategic counting, children in the LN group also showed poorer performance than their peers in the NA group. This was probably because the task was designed to tap children's place-value concept, which is the bridge between exact numerosities and symbolic numerals. To complete the task, children would need to have a sense of how many small squares there were in each item and then represent the exact numerosities in symbolic numerals. The inferior performance of children in the LN group was probably due to their difficulty in dealing with symbolic numerals (Iuculano et al., 2008).

While children in the DD group appeared to be outperformed by their peers on non-symbolic addition, such difference in performance did not reach statistical significance. One possible reason was that children in the DD group might compensate their deficit by falling back on one-to-one counting, whereas their peers, with an intact ability to subitize, could add up the total by forming small groups. If that was the case, the inferiority of the DD group should be reflected by a slower response time when compared with their peers. Future studies might also compare the response time of the children to obtain a better picture of their ability levels. 


\subsection{Underlying Deficits of Dyscalculia and Low Numeracy}

In general, the present findings suggested that Chinese children with dyscalculia performed at normal level in symbolic tasks, but failed in non-symbolic tasks. By contrast, those with low numeracy performed at normal level in non-symbolic tasks, but failed in symbolic tasks. Such findings were consistent with the previous proposals, based on studies with Western children, suggesting that dyscalculia was caused by a deficit in processing exact non-symbolic numerosities (Butterworth, 2005a), whereas low numeracy was caused by a deficit in understanding symbolic numerals (Iuculano et al., 2008). The core deficits of dyscalculia and low numeracy, therefore, appeared to be universal across number-naming systems and cultures.

Although the first-graders with dyscalculia or low numeracy in our study improved in the tasks associated with their respective core deficits, their performance tended to remain lagging behind that of their peers. This suggested that their core deficits were persistent, and that everyday mathematical learning at school was not sufficient to address the root of their problems. While some of them managed to catch up with their counterparts probably by avoiding their deficit areas and by falling back on primitive counting strategy or even rote-learning, they were likely to encounter difficulties as they proceeded to more advanced mathematical learning, which demands a genuine understanding of numeracy. Hence, early identification for additional learning support is the ultimate key to help these children in the 
long run.

\subsection{Implications for Screening and Learning Support}

The present findings point to a promising direction for developing a screening tool for mathematical difficulties among Chinese children. Evaluation of children's symbolic and non-symbolic numerical performance proved to be an informative approach to reveal their core deficits in mathematical learning, thereby facilitating the screening of, and differentiation between, dyscalculia and low numeracy. In the educational settings, teachers can examine and compare the symbolic (e.g., computation with numerals) and non-symbolic (e.g., computation with concrete manipulatives) performance of children who struggle with mathematics, to gain a better understanding of their underlying difficulties. Both symbolic and non-symbolic tasks compatible with the local mathematics curriculum (Gliga \& Gliga, 2012) should also be included in the future development of a formal screening instrument. Depending on their core deficits, these children should be provided with specific additional learning support as early as possible. In particular, children with dyscalculia are in need of training in the concept of exact numerosities, whereas those with low numeracy may find training in understanding symbolic numerals more beneficial.

\section{Conclusions}

This study was the first to explore developmental dyscalculia and low numeracy among 
Chinese children. By examining first-graders' performance in various symbolic and non-symbolic numerical tasks, we showed that children with dyscalculia demonstrated impaired performance in non-symbolic tasks but not symbolic ones, whereas the opposite was the case for those with low numeracy. These findings went beyond prior research which documented only general mathematical performance deficits for these two groups of children. The present findings suggested that dyscalculia had its root primarily in problematic concept of exact non-symbolic numerosities, whereas low numeracy's underlying deficit was related to a poor understanding of symbolic numerals. These findings not only inform researchers and educators on the roots of these two kinds of deficits, but also suggest a direction for the screening of, and designing learning support for, Chinese children with different core deficits in mathematical learning. 


\section{Acknowledgements}

We thank the staff at the Educational Psychology Services (New Territories) Section of Hong

Kong Education Bureau for their logistic support in this research. 


\section{References}

Chan, W. W. L., Au, T. K., \& Tang, J. (under review). Strategic counting: A novel assessment of place-value understanding. Learning and Instruction.

Butterworth, B. (1999). The mathematical brain. London: Macmillan.

Butterworth, B. (2003). Dyscalculia Screener. London: nfer Nelson Publishing Company Ltd.

Butterworth, B. (2005a). Developmental dyscalculia. In J. I. D. Campbell (Ed.), Handbook of mathematical cognition (pp. 455-467). New York: Psychology Press.

Butterworth, B. (2005b). The development of arithmetical abilities. Journal of Child Psychology and Psychiatry, 46, 3-18.

Butterworth, B., Cipolotti, L., \& Warrington, E. K. (1996). Short-term memory impairments and arithmetical ability. Quarterly Journal of Experimental Psychology, 49A, 251-262.

Butterworth, B., \& Reigosa-Crespo, V. (2007). Information processing deficits in dyscalculia. In D.B. Berch \& M.M.M. Mazzocco (Eds.), Why is math so hard for some children? The nature and origins of mathematical learning difficulties and disabilities (pp. 6581). Baltimore, MD: Paul H. Brookes Publishing Co.

Cohen, L., Dehaene, S., Cochon, F., Lehericy, S., \& Naccache, L. (2000). Language and calculation within the parietal lobe: A combined cognitive, anatomical and fMRI study. Neuropsychologia, 38, 1426-1440.

Geary, D. C. (1993). Mathematical disabilities: Cognition, neuropsychological and genetic 
components. Psychological Bulletin, 114, 345-362.

Geary, D. C., \& Hoard, M. K. (2001). Numerical and arithmetical deficits in learning-disabled children: Relation to dyscalculia and dyslexia. Aphasiology, 15, 635-647.

Gliga, F., \& Gliga, T. (2012). Romanian screening instrument for dyscalculia. ProcediaSocial and Behavioral Sciences, 33, 15-19.

Hong Kong Curriculum Development Council. (2000). Mathematics Curriculum Guide P1 P6. Hong Kong SAR Government.

Huntsinger, C. S., Jose, P. E., Liaw, F. R., \& Ching, W. (1997). Cultural differences in early mathematics learning: A comparison of Euro-American, Chinese-American, and Taiwan-Chinese families. International Journal of Behavioral Development, 21, 371-388.

Iuculano, T., Tang, J., Hall, C. W. B., \& Butterworth, B. (2008). Core information processing deficits in developmental dyscalculia and low numeracy. Developmental Science, 11, 669-680.

Jordan, N., Hanich, L. B., \& Kaplan, D. (2003). Arithmetic fact mastery in young children: A longitudinal investigation. Journal of Experimental Child Psychology, 85, 103-119.

Landerl, K., Bevan, A., \& Butterworth, B. (2004). Developmental dyscalculia and basic numerical capacities: a study of 8-9-year-old students. Cognition, 93, 99-125.

Miller, K. F., Kelly, M., \& Zhou, X. (2005). Learning mathematics in China and the United 
States. In J. I. D. Campbell (Ed.), Handbook of mathematical cognition (pp. 163-178).

New York: Psychology Press.

Miller, K. F., Smith, C. M., Zhu, J., \& Zhang, H. (1995). Preschool origins of cross-national differences in mathematical competence: The role of number-naming systems. Psychological Science, 6, 56-60.

Miller, K. F., \& Stigler, J. W., (1987). Counting in Chinese: Cultural variation in a basic cognitive skill. Cognitive Development, 2, 279-305.

Rousselle, L., \& Noël, M.P. (2007). Basic numerical skills in children with mathematics learning disabilities: a comparison of symbolic vs. non-symbolic number magnitude processing. Cognition, 102, 361-395.

Saxton, M., \& Cakir, K. (2006). Counting-on, trading, and partitioning: Effects of training and prior knowledge on performance on base-10 tasks. Child Development, 77, 767-785.

Shalev, R. S. (2007). Prevalence of developmental dyscalculia. In D. B. Berch \& M. M. M. Mazzocco (Eds.), Why is math so hard for some children? The nature and origins of mathematical learning difficulties and disabilities (pp. 49-60). Baltimore, MD: Paul H. Brooks Publishing Co.

Shalev, R. S., \& Gross-Tsur, V. (2001). Developmental dyscalculia. Pediatric Neurology, 24, $337-342$.

Starkey, P., \& Cooper, R.G., Jr. (1980). Perception of numbers by human infants. Science, 210, 
1033-1035.

Starkey, P., \& Klein, A. (2008). Sociocultural influences on young children's mathematical knowledge. In O. N. Saracho \& B. Spodek (Ed.), Contemporary perspectives on mathematics in early childhood education (pp. 45-66). Baltimore. MD: IAP, INC.

Starkey, P., Spelke, E. S., \& Gelman, R. (1990). Numerical abstraction by human infants. Cognition, 36, 97-128.

Tang, J. (2010). Developing a web-based testing platform for the identification of developmental dyscalculia. Chinese Medical Journal, 123, 283.

Wynn, K. (1992). Addition and subtraction by human infants. Nature, 358, 749-751.

Wynn, K. (1995). The origins of numerical knowledge. Mathematical Cognition, 1, 35-60.

Zhou, X., Huang, J., Wang, Z., Wang, B., Zhao, Z., Yang, L., \& Zheng-zheng, Y. (2006). Parent-child interaction and children's number learning. Early Child Development and Care, 176, 763-775. 
Figure 1. Flow Chart of Classification of Children: DD, LN, NA

Figure 2a. Performance in Count Sequence (Symbolic Task, Max. Score =65).

Figure 2b. Performance in Reading Aloud Arabic Numbers (Symbolic Task, Max.

Score $=8)$.

Figure 2c. Performance in Reading Aloud Chinese Numbers (Symbolic Task, Max.

Score $=8)$.

Figure 2d. Performance in Symbolic Addition (Symbolic Task, Max. Score = 8).

Figure 2e. Performance in Grouping-In-Ten (Non-Symbolic Task, Max. Score =4).

Figure 2f. Performance in Place-Value Representation (Non-Symbolic Task, Max.

Score $=8)$.

Figure 2g. Performance in Strategic Counting (Non-Symbolic Task, Max. Score = 10).

Figure 2h. Performance in Non-symbolic Addition (Non-Symbolic Task, Max. Score $=4)$. 
Figure 1.

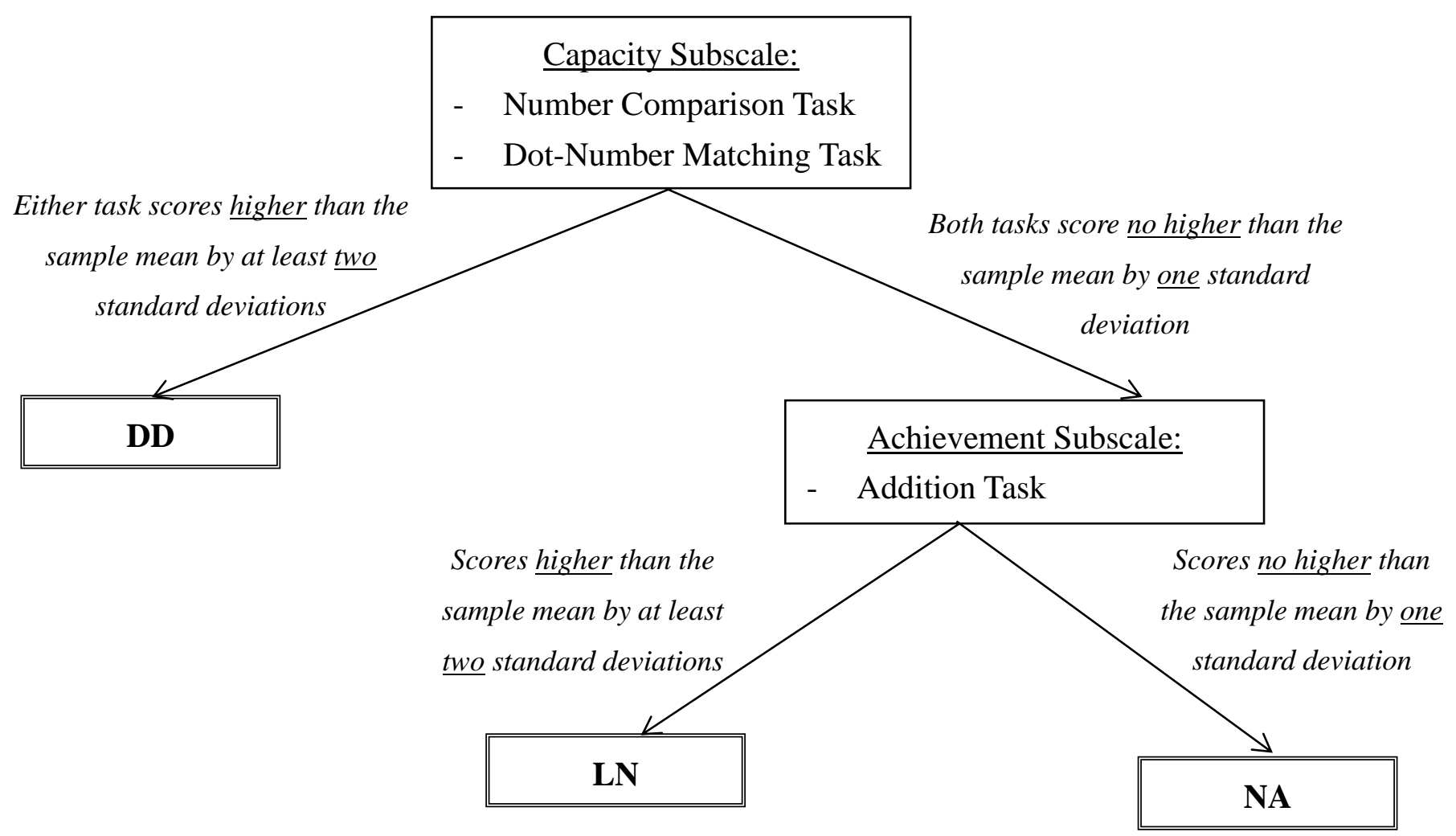


Figure 2a.

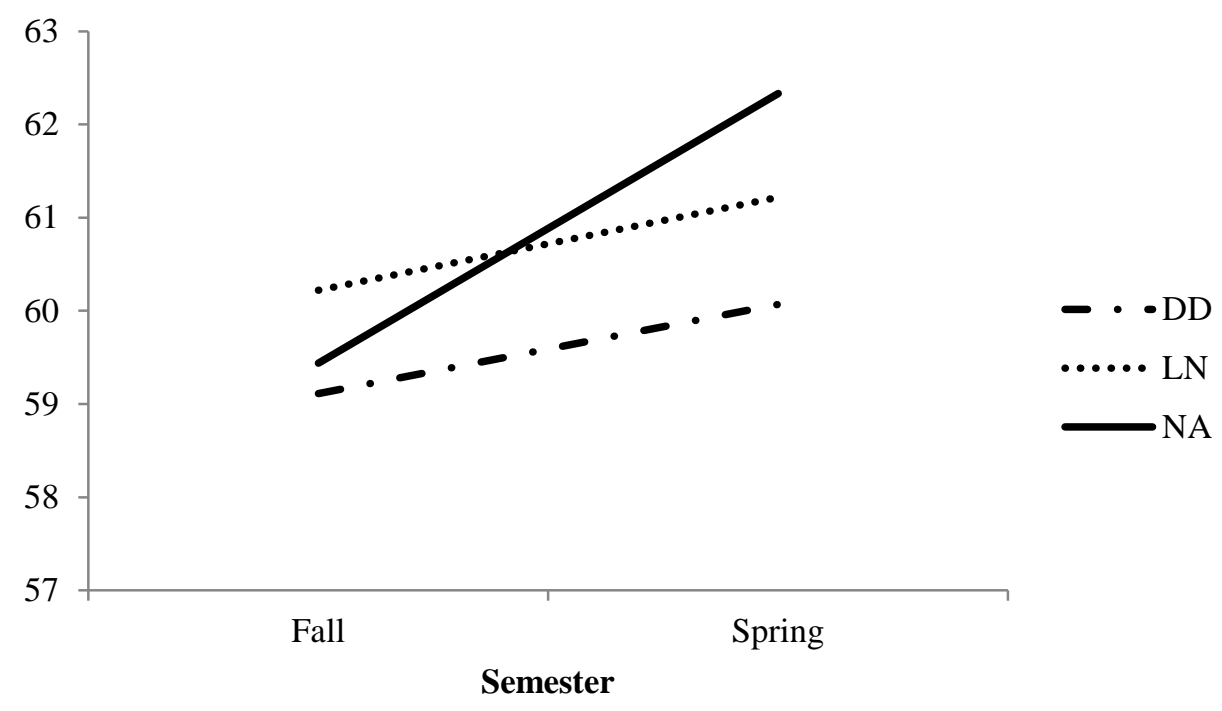


Figure 2b.

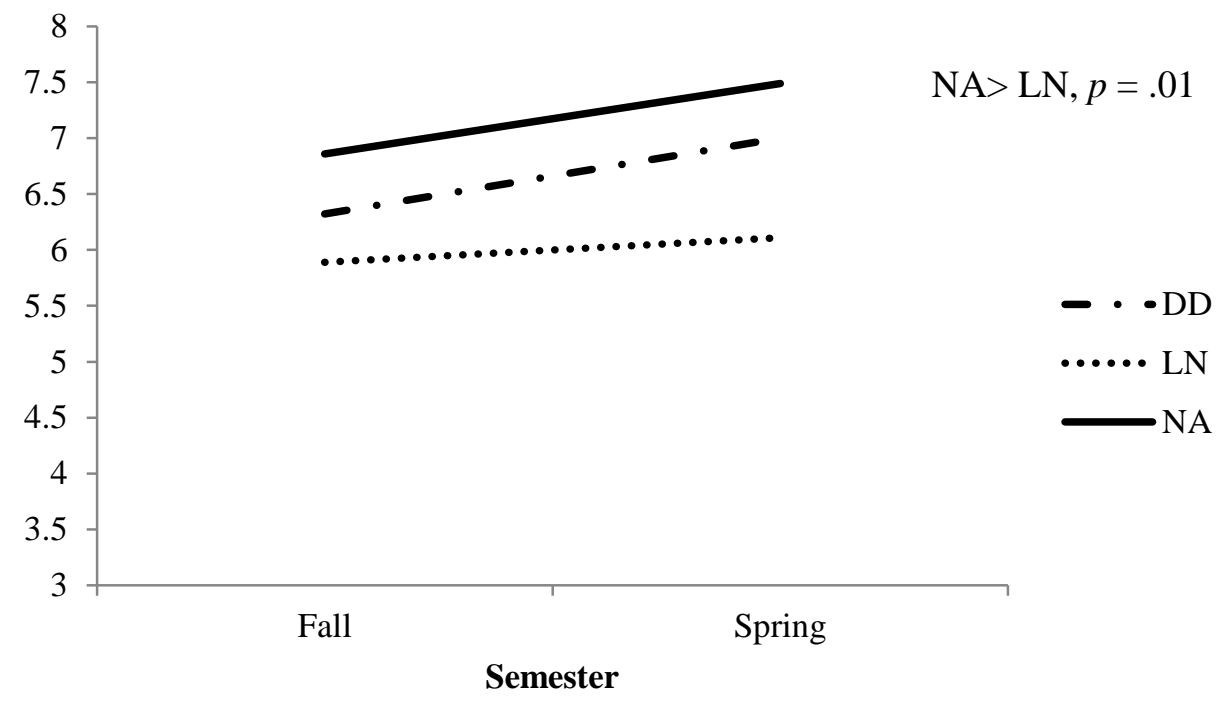


Figure 2c.

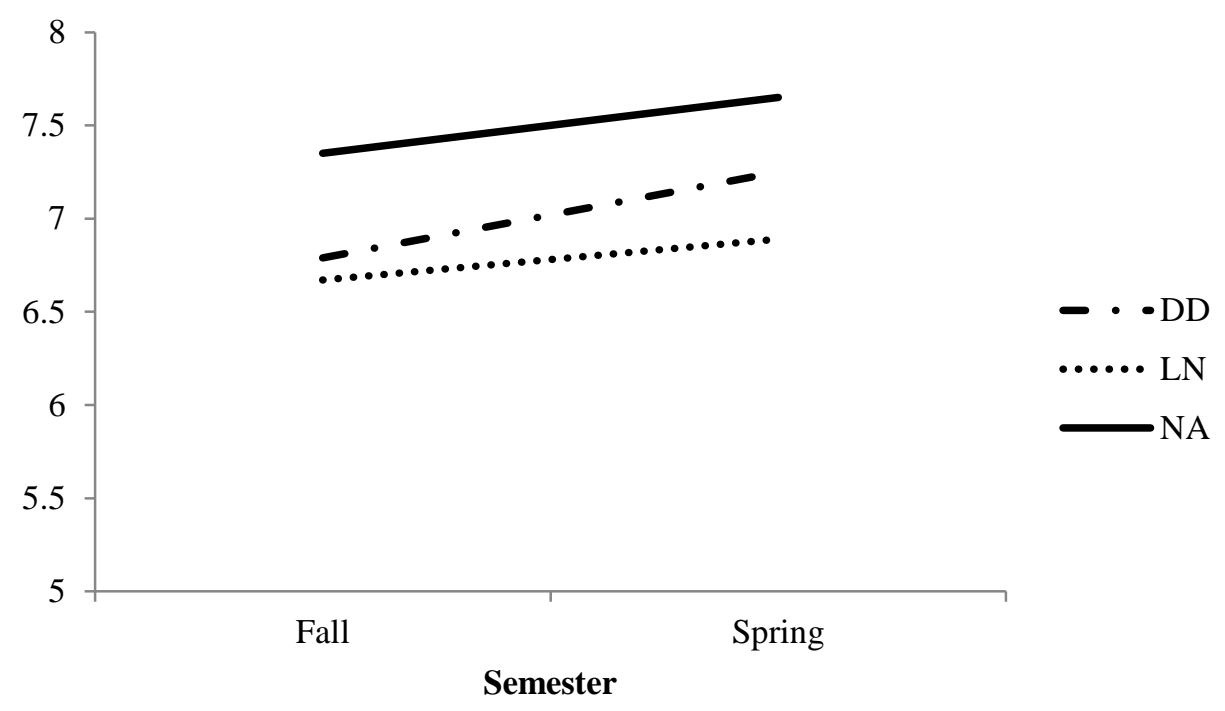


Figure 2d.

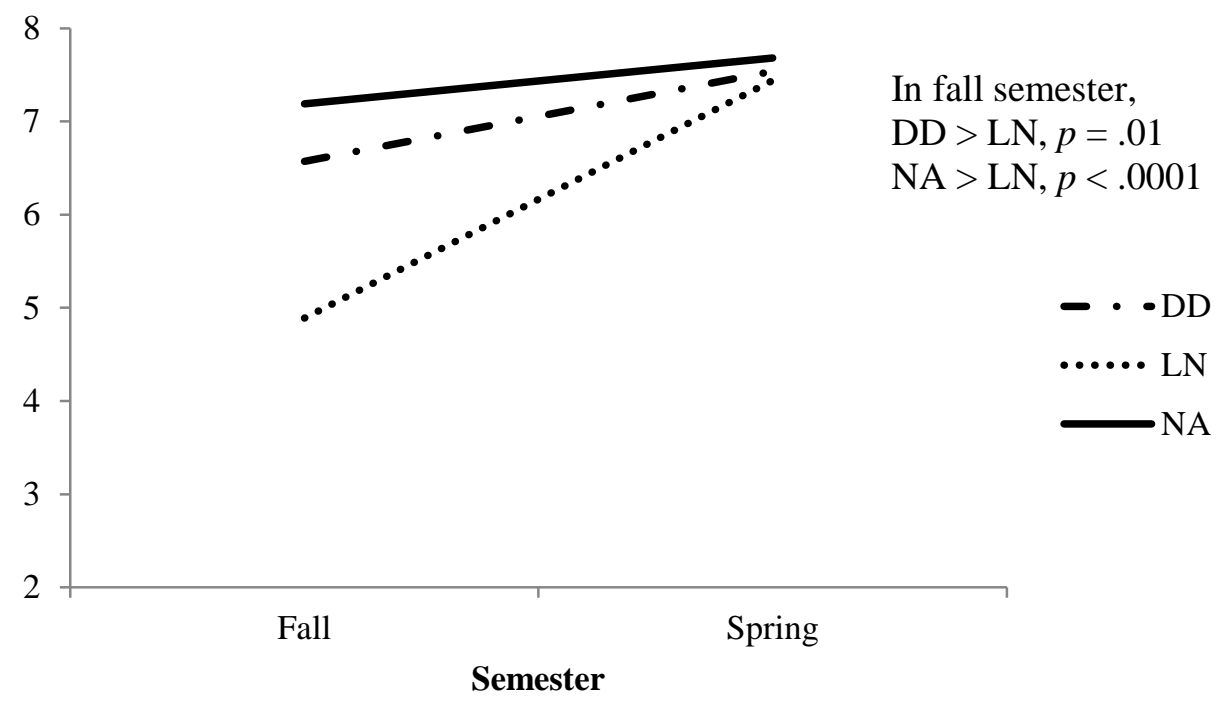


Figure 2e.

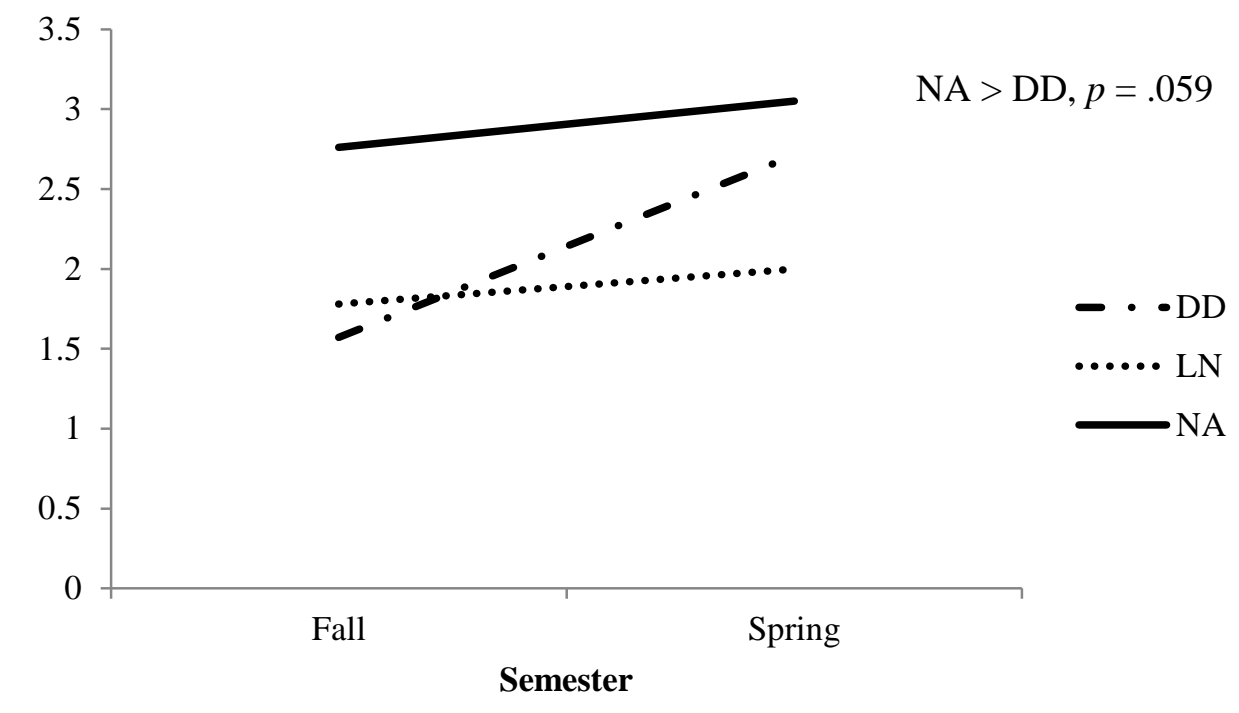


Figure 2f.

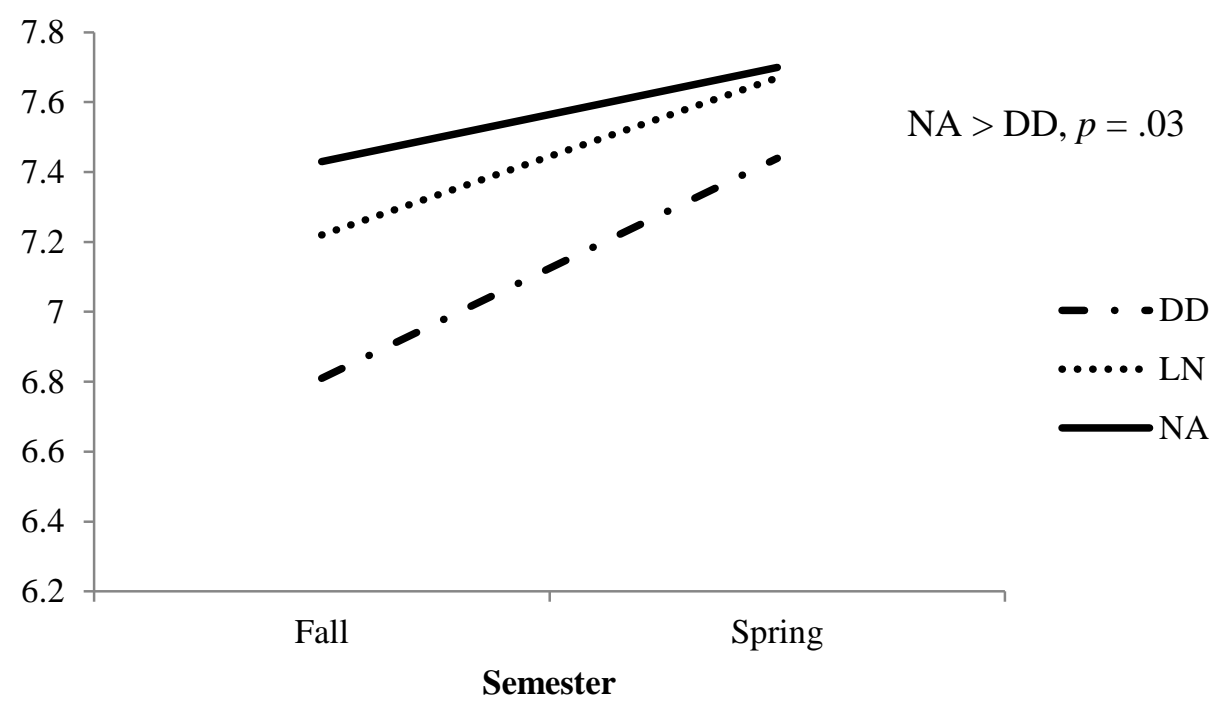


Figure 2g.

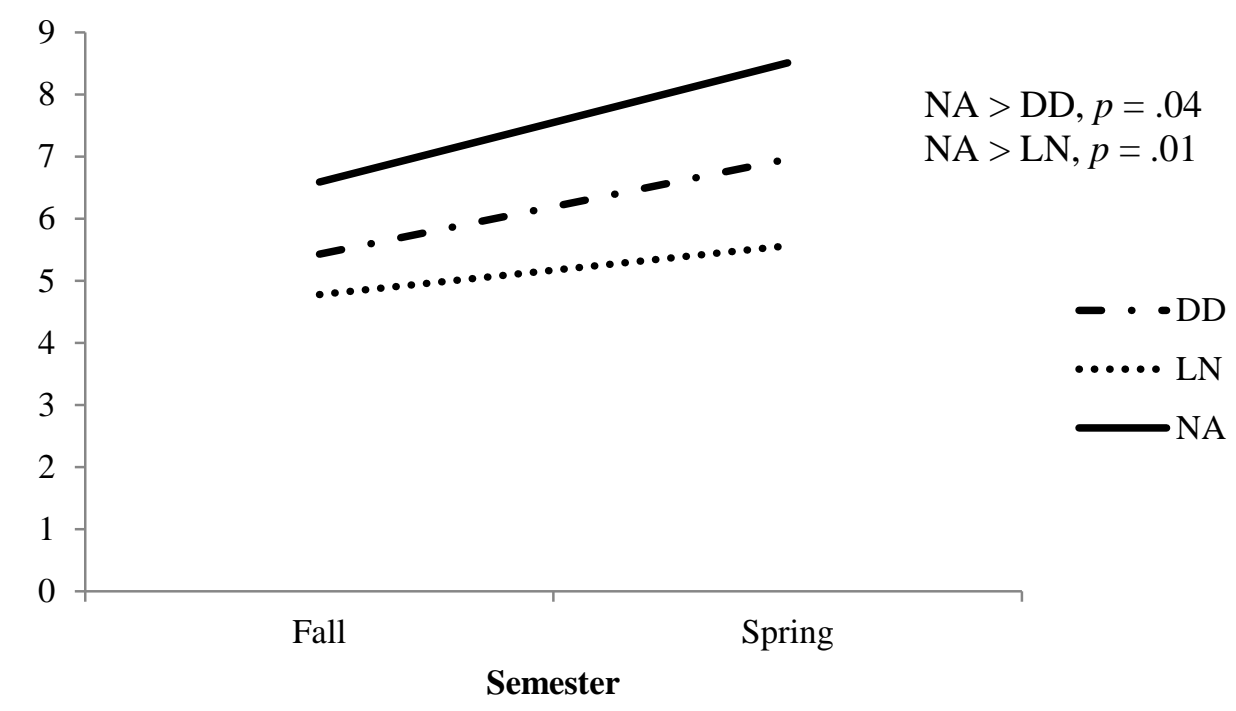

$\mathrm{NA}>\mathrm{DD}, p=.04$

$\mathrm{NA}>\mathrm{LN}, p=.01$

$-\cdot-\mathrm{DD}$

N

emester 
Figure $2 \mathrm{~h}$.

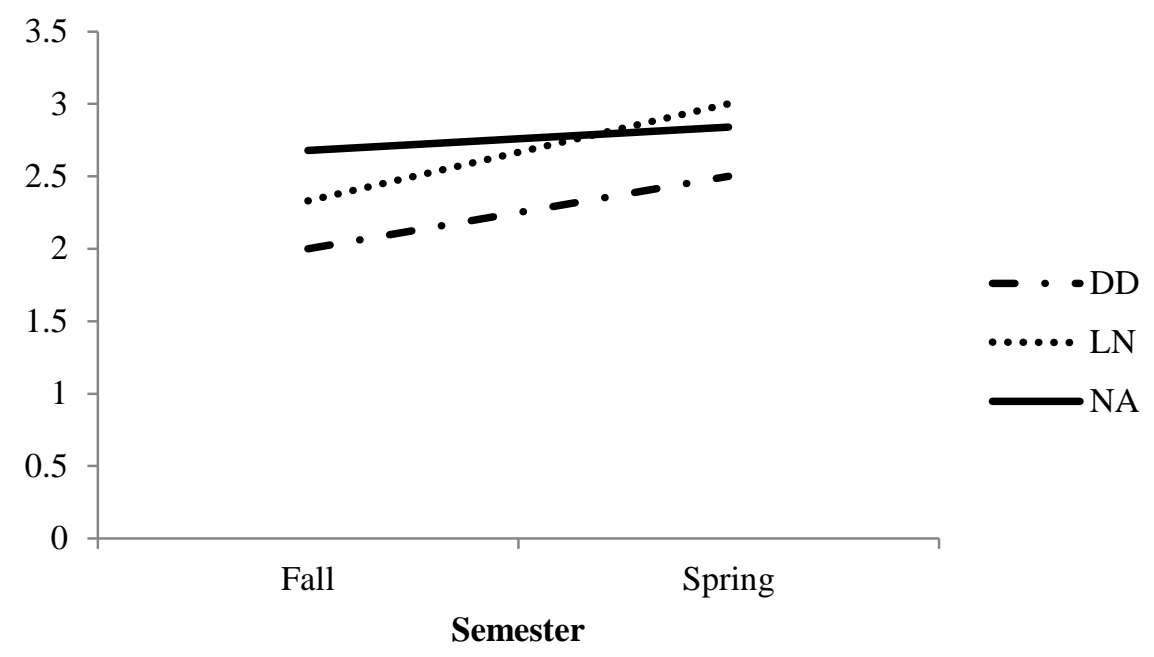


Table 1. Descriptive Information of Children in the Three Groups.

\begin{tabular}{lccc}
\hline & $\begin{array}{c}\text { Developmental } \\
\text { Dsycalculia (DD) } \\
\text { Group }(N=28)\end{array}$ & $\begin{array}{c}\text { Low Numeracy (LN) } \\
\text { Group }(N=9)\end{array}$ & $\begin{array}{c}\text { Normal Achievement } \\
\text { (NA) Group }(N=37)\end{array}$ \\
\hline Gender & & & \\
Male & 20 & 3 & 26 \\
Female & 8 & $77.3(3.2)$ & 11 \\
Age (months) & $78.8(4.2)$ & $111.3(12.7)$ & $109.0(4.4)$ \\
Nonverbal IQ & $109.5(13.5)$ & & $11.0)$ \\
\hline
\end{tabular}

Note. Standard deviations are in parentheses. 